\title{
NECESSIDADES PESSOAIS DE ENFERMEIROS DURANTE A PANDEMIA DA COVID-19 EM MATO GROSSO
}

Vagner Ferreira do Nascimento ${ }^{1}$ Thalise Yuri Hattori ${ }^{1}$

Ana Cláudia Pereira Terças Trettel ${ }^{1,2}$

\author{
https://orcid.org/0000-0002-3355-163X \\ http://orcid.org/0000-0003-4491-0375 \\ https://orcid.org/0000-0002-1878-2237
}

Objetivo: Identificar as necessidades pessoais de Enfermeiros durante a pandemia da COVID-19 em Mato Grosso. Método: estudo exploratório e qualitativo, realizado em abril de 2020, com Enfermeiros do estado de Mato Grosso. Utilizou-se o discurso do sujeito coletivo e a Teoria das Necessidades Humanas de Maslow. Resultados: Os participantes apresentaram necessidades intermediárias (segurança e sociais) quanto as categorias da teoria de Maslow. Em relação aos discursos foram obtidas quatro ideias centrais: autocuidado, necessidade de afeto, compreensão do distanciamento e prejuizos na relação conjugal. Conclusões: As necessidades pessoais dos Enfermeiros refletem o impacto das medidas protetivas recomendadas durante a pandemia da COVID-19 tanto no contexto laboral como familiar. Descritores: Pandemias; Coronavirus; Pessoal de Saúde; Carência Psicossocial.

\section{PERSONAL NEEDS OF NURSES DURING THE COVID-19 PANDEMIC IN MATO GROSSO}

Objective: To identify the personal needs of Nurses during the COVID-19 pandemic in Mato Grosso. Method: exploratory and qualitative study, conducted in April 2020, with Nurses from the state of Mato Grosso. The collective subject discourse and Maslow Theory of Human Needs were used. Results: The participants presented intermediate needs (security and social) regarding the categories of Maslow theory. Regarding the speeches, four central ideas were obtained: self-care, need for affection, understanding of distance and losses in the marital relationship. Conclusions: Nurse personal needs reflect the impact of the protective measures recommended during the COVID-19 pandemic in both the work and family context. Descriptors: Pandemics; Coronavirus; Health Personnel; Psychosocial Deprivation.

\section{NECESIDADES PERSONALES DE ENFERMERAS DURANTE LA PANDEMIA DA COVID-19 EM MATO GROSSO}

Objetivo: identificar las necesidades personales de las Enfermeras durante la pandemia da COVID-19 en Mato Grosso. Metodo: estudio exploratorio y cualitativo, realizado en abril de 2020, con Enfermeras del estado de Mato Grosso. Se utilizó el discurso del sujeto colectivo y la Teoría de las Necesidades Humanas de Maslow. Resultados: Los participantes presentaron necesidades intermedias (seguridady sociales) en relación con las categorías de la teoría de Maslow. Con respecto a los discursos, se obtuvieron cuatroideas centrales: autocuidado, necesidad de afecto, comprensión de la distancia y pérdidas en la relación matrimonial. Conclusiones: Las necesidades personales de las Enfermeras reflejan el impacto de las medidas de protección recomendadas durante la pandemia da COVID-19, tanto en el contexto laboral comofamiliar. Descriptores: Pandemias; Coronavirus; Personal de Salud; Carencia Psicosocial.

${ }^{1}$ Universidade do Estado de Mato Grosso, campus de Tangará da Serra, MT.

2 Universidade Federal de Mato Grosso, campus de Cuiabá, MT.

Autor Correspondente: Ana Cláudia Pereira Terças Trettel. E-mail: ana.claudia@unemat.br

Recebido: 29/4/2020 - Aceito : 26/5/2020 


\section{INTRODUÇÃO}

O avanço da pandemia do novo coronavirus (COVID-19) no mundo está relacionado ao aumento das viagens, somado à integração global, urbanização e maior exploração do ambiente natural ${ }^{(1)}$. Uma preocupação importante nesse cenário, consiste na capacidade da alta transmissibilidade do patógeno já que a sua infectividade em oligossintomáticos ou assintomáticos é superior às epidemias anteriores originadas por outros coronavírus ${ }^{(2)}$.

Em uma situação de emergência de saúde pública, os enfermeiros, profissionais que estão na linha de frente, apresentam uma maior exposição aos fatores de risco ${ }^{3}$. Especificamente em situações de desastre/calamidade como nessa pandemia, os enfermeiros normalmente sublimam suas próprias necessidades para atuarem ativamente nos cuidados em saúde e trabalhos humanitários. Suas contribuições altruistas são direcionadas pela responsabilidade moral, ética e profissional, sobretudo em defesa e proteção à vida ${ }^{(3-5)}$

O negligenciamento da saúde dos enfermeiros, quer seja no processo de gerenciamento das equipes e escalas, como da disponibilidade de infraestrutura e condições de trabalho adequado e atenção das instituições e gestores, aumentam as chances de adoecimento(6-10). Pesquisa realizada em Henan, revelou que entre Enfermeiros a presença de emoções negativas e positivas se entrelaçaram e coexistiram no transcorrer da pandemia da COVID19, porém prevaleceu e acentuou-se as negativas ${ }^{(11)}$.

Apesar de estudos enfatizarem à saúde mental dos profissionais durante a pandemia ${ }^{(11-14)}$, no Brasil e particularmente no estado de Mato Grosso, ainda não se conhece as necessidades dos enfermeiros. Assim, este estudo objetivou identificar as necessidades pessoais de enfermeiros durante a pandemia da COVID-19 em Mato Grosso.

\section{MÉTODO}

\section{Tipo de estudo}

Estudo exploratório e qualitativo, seguindo o protocolo internacional Consolidated Criteria For Reporting Qualitative Research (COREQ).

\section{Local do Estudo}

Realizado no estado de Mato Grosso, Brasil. A escolha desse estado ocorreu por ser o terceiro maior em extensão do país e o maior do Centro-Oeste, possuir população quilombola, ribeirinha, assentados, garimpeiros e a maior diversidade étnica de indígenas do Brasil, o que amplia os desafios no cuidar em saúde.

\section{Participantes do Estudo}

A população foi composta por enfermeiros atuantes em instituições de saúde. A amostragem foi do tipo não probabilística, intencional, definido pela saturação de dados, até atingir o objetivo do estudo. Foram inclusos no estudo, enfermeiros com atuação mínima de 12 meses e com vínculo ativo na Enfermagem. Excluiu-se, enfermeiros com idade superior a 60 anos por comporem grupo de maior risco para infecção da COVID-19. Totalizou-se 22 enfermeiros no estudo, sem desistências.

\section{Procedimentos de Coleta de dados}

$\mathrm{O}$ estudo foi realizado em abril de 2020. Os enfermeiros foram convidados a participarem do estudo, via contato telefônico, disponibilizado pelos serviços de saúde que atuam. Após esse contato, aqueles que aceitaram participar, receberam individualmente, através do aplicativo WhatsApp uma questão norteadora: "Quais suas necessidades pessoais, nesse momento de pandemia da COVID-19". Os enfermeiros tiveram 24 horas para realizarem a devolutiva, através de mensagem de texto, pelo próprio aplicativo.

\section{Procedimentos de análise e tratamento dos dados}

Para organização e análise dos dados, utilizou-se o discurso do sujeito coletivo (DSC) e a Teoria das Necessidades Humanas de Maslow ${ }^{(15)}$. No DSC, seguiu-se as quatro etapas operacionais: seleção das expressões-chave $(\mathrm{ECH})$ de cada discurso, podendo ser contínuas ou descontínuas; identificação da ideia central (IC) de cada uma dessas $\mathrm{ECH}$; identificação das IC semelhantes ou complementares; junção das ECH referentes às IC, formando uma síntese do discurso ${ }^{(16)}$.

A Teoria de Maslow, subdivide em necessidades: fisiológicas (sono, alimentação, desejos sexuais, respiração, água, excreção), de segurança (segurança do corpo, do emprego, de recursos, de moralidade, da família, de saúde, da propriedade), sociais (troca afetiva, associações, aceitação, intimidade sexual, amizades, família), de estima (valorização, autoestima, respeito, confiança, conquista) e de realização (autorrealização, moralidade, criatividade, espontaneidade, solução de problemas, ausência de preconceito, aceitação dos fatos) ${ }^{(15)}$.

\section{Aspectos éticos}

Este estudo respeitou todos aspectos éticos em pesquisa, conforme a Resolução 466/2012 do Conselho Nacional de Saúde (CNS), com Certificado de Apresentação para Apreciação Ética (CAAE) n. 28214720.9.0000.5166 e parecer de aprovação n. 3.903.714/2020. 


\section{RESULTADOS}

Em relação as categorias da teoria de Maslow, os enfermeiros do estudo nesse momento pandêmico possuem necessidades intermediárias (segurança e sociais). Apesar de haver DSC de enfermeiros apontando a valorização dos cuidados para manter a integridade da saúde, a maioria reconhece que suas necessidades atuais estão associadas aos impactos causados no contexto familiar e nas relações de amor e afeto entre seus entes queridos, conforme o Quadro 1.

Quadro 1. Discursos dos 22 enfermeiros de Mato Grosso, Brasil sobre suas necessidades pessoais na pandemia da COVID-19, em abril de 2020, conforme as categorias de Maslow.

\begin{tabular}{|c|c|c|c|c|}
\hline \multicolumn{5}{|c|}{ DSC } \\
\hline $\begin{array}{c}\text { Fisio- } \\
\text { lógi- } \\
\text { cas }\end{array}$ & Segurança & Sociais & $\begin{array}{c}\text { Esti- } \\
\text { ma }\end{array}$ & $\begin{array}{c}\text { Rea- } \\
\text { liza- } \\
\text { ção }\end{array}$ \\
\hline - & $\begin{array}{l}\text { Acho que a mi- } \\
\text { nha necessidade } \\
\text { é igual a todos os } \\
\text { colegas de traba- } \\
\text { lho, é o autocui- } \\
\text { dado ativo e per- } \\
\text { manentes, para } \\
\text { não se infectar, } \\
\text { não adoecer e } \\
\text { acabar conta- } \\
\text { minando outras } \\
\text { pessoas. E so- } \\
\text { bretudo cuidar de } \\
\text { mim e dos meus } \\
\text { colegas. Há uma } \\
\text { ansiedade em } \\
\text { manter esse au- } \\
\text { tocuidado. ECH } \\
\text { (n=7) }\end{array}$ & 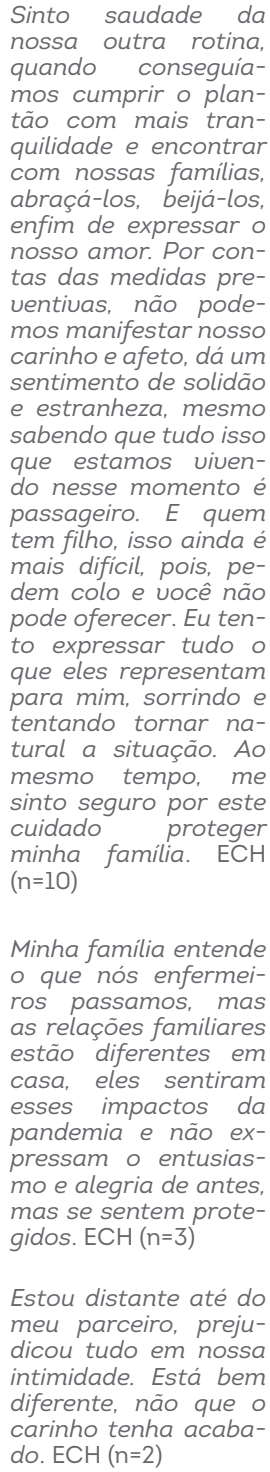 & - & - \\
\hline
\end{tabular}

Os discursos dos enfermeiros do estudo, concentraram em quatro IC que representam alguns elementos presentes nos DSC e que englobam as categorias de segurança e sociais. Entre as IC, a categoria de necessidades sociais, abrangem a quantidade maior de demandas para esses profissionais, de acordo com o Quadro 2.

Quadro 2. Ideias centrais e elementos das categorias de Maslow presentes nos DSC dos 22 enfermeiros, em abril de 2019, em Mato Grosso, Brasil.

\begin{tabular}{|c|c|c|}
\hline \multirow{4}{*}{ IC } & Segurança & Sociais \\
\hline & \multirow{3}{*}{$\begin{array}{l}\text { IC } 1 \text { - Autocuidado } \\
\text { (segurança do corpo, } \\
\text { proteção e saúde) }\end{array}$} & $\begin{array}{l}\text { IC } 2 \text { - Necessidade de afeto } \\
\text { (troca afetiva e familia) }\end{array}$ \\
\hline & & $\begin{array}{l}\text { IC } 3 \text { - Compreensão do distancia- } \\
\text { mento } \\
\text { (aceitação) }\end{array}$ \\
\hline & & $\begin{array}{l}\text { IC } 4 \text { - Prejuizos na relação conjugal } \\
\text { (intimidade sexual) }\end{array}$ \\
\hline
\end{tabular}

\section{DISCUSSÃO}

Um dos possíveis motivos para que os enfermeiros possuam necessidades pessoais mais próximas das categorias basilares da teoria de Maslow pode se relacionar à fase da pandemia no estado de Mato Grosso, esta que não alcançou a estabilização, e consequentemente, as exigências de cuidados de prevenção são prioritárias, refletindo em maiores demandas para o autogerenciamento do profissional.

Nesse período, em que a pandemia está em crescimento exponencial, existe apreensão entre os profissionais devido à indisponibilidade de medidas básicas de proteção. Além disso, a necessidade do rigor de estratégicas de segurança em meio a exposição constante ao vírus é uma grande preocupação(17). Como apontado entre os enfermeiros do estudo, há ansiedade para manterem o cuidado com a prevenção individual e coletiva, ademais, esse comportamento, somado à extenuação laboral se tornam mecanismos de riscos para a infecção, pela interferência da autocobrança ${ }^{(18-19)}$.

Para tanto, o autocuidado é um importante recurso para o profissional da linha de frente, principalmente o Enfermeiro, pois ações individuais que promovam o bloqueio do novo coronavírus como, por exemplo, a adesão de práticas baseadas em evidências e a consciência que o ambiente laboral coincide com local potencial para a infecção, que é compartilhado com a equipe, refletem em atitudes coletivas que tendem a suprir as necessidades de segurança diante da COVID-19(20). 
Na China, além das necessidades de segurança, as necessidades sociais, integraram o grupo das principais demandas apontadas por enfermeiros, particularmente expressas nas relações com a equipe de saúde e familiares ${ }^{(21)}$. Embora as necessidades humanas de Maslow sigam uma hierarquia piramidal, das mais básicas (fisiológicas) à mais elevadas (estima e realização), é importante entender que durante a ocorrência de problemas de grande magnitude, mesmo se as necessidades de segurança ainda não estiverem atendidas, as necessidades sociais que remetem neste caso ao apoio de familiares e amigos são manifestadas ${ }^{(22)}$, como observado no presente estudo.

As necessidades sociais dos enfermeiros durante a pandemia foram associadas ao distanciamento social, manutenção da quarentena e sensação de desamparo. Essas circunstâncias trazem prejuízos para a dinâmica familiar, principalmente no que se diz respeito às trocas de afeto não ocorridas como desejadas, e que nesse período, apresentam-se mais intensas do que no cotidiano(23).

Estar próximo da família e ser acolhido são fatores motivacionais positivos para os enfermeiros nesse período de pandemia. No entanto, mudanças na rotina como não poder voltar para casa ou protelar/restringir o contato direto e afetivo com os seus familiares, causa tristeza e frustação. Essas sensações, ocorrem especialmente por serem impedidos de desenvolverem o que aprenderam na formação na Enfermagem, em serem referências de cuidado, com o estabelecimento de vínculos e estreitamento de distâncias. Diante disso, é importante que o profissional receba apoio psicoemocional, para lhe ajudar a compreender melhor a situação e permitir o reconhecimento dos riscos do seu trabalho como parte da sua profissão, e utilizar essa vivência para ressignificar as medidas restritivas ${ }^{(11,24-25)}$

Essa ressignificação alcança os familiares, pois, apesar dos sinais de tristeza passam a entender a ausência de afeto e distanciamento social, como parte do cuidado do seu ente querido e percebem como sentimento de proteção(24,26). Esse cuidado dos Enfermeiros mato-grossenses, relaciona-se ao entendimento que a transmissão viral de humano para humano ocorre principalmente por contato próximo e direto(27-30) consequentemente, a disseminação da doença para os membros da família pode ser mais frequente para os casos de profissionais que estão na linha de frente nos serviços de saúde ${ }^{(26)}$.

Outras inquietações identificadas nos discursos dos Enfermeiros se referem ao distanciamento às crianças no contexto familiar. Apesar delas apresentarem menor grau de gravidade à infecção, a suscetibilidade para o contágio e alta possibilidade de transmissibilidade, pela dinâmica e natureza do intenso contato afetivo, há exigência de medidas protetivas. E para suprir as necessidades sociais dos familiares e das crianças diante dessas medidas, pesquisadora americana apontou algumas estratégias tecnológicas como, brincadeiras por telefonemas e videoconferências e jogo lúdicos para manter a sensação de controle, ensinando o que pode ser feito para eliminar o vírus e proteger quem se ama(31).

Nesta pesquisa, observou-se ainda que as relações conjugais dos Enfermeiros também foram afetadas e já indicam a sensação de perda da intimidade sexual. Ainda que não haja um consenso na literatura científica sobre a infecção pelo contato sexual, há recomendação que as práticas sexuais durante a pandemia da COVID-19 devem ser desencorajadas, pela possibilidade de transmissão através dos fluidos, principalmente pela saliva e via oral-fecal ${ }^{(21,32)}$. De todo modo, a relação sexual envolve um contato direto que inevitavelmente expõe ao risco de contágio(33)

Entre os enfermeiros do estudo, parece que essa necessidade se apoia mais ao aspecto de carência do parceiro afetivo, o desejo em compartilhar momentos, aconchego e descompressão conjunta, porém impedidos pelo distanciamento social e nova rotina assumida ${ }^{(24)}$. Um dos motivos para essa necessidade pode se relacionar ao perfil de qualidade de vida de enfermeiros brasileiros, que possuem pouco tempo para lazer(34), utilizando as vivências do lar como seus momentos de reencontro e descanso. Ao contrário, segundo a literatura, para outros casais, manterem-se em quarentena juntos nesta pandemia está sendo um motivo de sofrimento e violência, pelos conflitos e comportamentos nocivos gerados, estando protegidos do vírus, porém em risco de danos à integridade física e psicoemocional(35)

Estudo internacional sobre as necessidades de enfermeiros chineses durante a pandemia da COVID-19, revelou que além de necessidades sociais (apego às relações familiares) e de segurança (autocuidado com a saúde), possuem necessidades de crescimento(23), aspecto não observado neste estudo. Ainda assim, parece que as necessidades dos Enfermeiros do estudo são próximas de outras realidades e indicam a indissociabilidade da vida profissional com a vida pessoal desses profissionais, e o impacto direto da rotina de trabalho durante a pandemia no bem-estar individual, especialmente no âmbito familiar.

\section{Limitações do estudo}

As limitações do estudo estão relacionadas principalmente com a forma de coleta de dados, via aplicativo, que pode não ter abrangido todas as percepções do participante e/ou terem suavizado seus anseios atuais. Outra limita- 
ção, refere-se à percepção de necessidades humanas que podem variar por aspectos regionais e culturais, não podendo generalizar os achados do estudo.

\section{Contribuições para a prática}

O conhecimento das necessidades pessoais dos enfermeiros durante a pandemia da COVID-19 fornece elementos para subsidiar estratégias de proteção à saúde do trabalhador que podem influenciar positivamente na qualidade de vida desses profissionais e minimizar o impacto dos fatores estressantes que geram adoecimento e interferências das relações sociais. Além disso, poderá ainda contribuir com a compreensão de aspectos que determinam satisfação, sofrimento e a permanência/sustentação do enfermeiro no cotidiano assistencial.

\section{CONCLUSÃO}

Identificou-se que as necessidades pessoais dos enfermeiros, concentraram-se nas categorias de segurança e sociais da teoria de Maslow. As necessidades de segurança, voltou-se aos cuidados para evitar a infecção/contamina- ção com o vírus e as necessidades sociais se relacionaram especificamente ao impacto do distanciamento social no contexto domiciliar, com carência de afeto e mudanças na dinâmica familiar.

Apesar disso, os discursos revelaram necessidades pessoais semelhantes e que se cruzam, ao compreender que ambas geram sofrimentos e se referem à proteção individual e coletiva. Essas necessidades refletem o impacto das medidas protetivas recomendadas durante a pandemia da COVID-19 tanto no contexto laboral como familiar.

\section{CONTRIBUIÇÕES DOS AUTORES}

VFN: concepção e/ou desenho do estudo, coleta, análise e interpretação dos dados, redação e revisão crítica do manuscrito e aprovação da versão final a ser publicada: TYH: análise e interpretação dos dados, redação e revisão crítica do manuscrito e aprovação da versão final a ser publicada; ACPTT: coleta, análise e interpretação dos dados, redação e revisão crítica do manuscrito e aprovação da versão final a ser publicada.

\section{REFERÊNCIAS}

1. Gatti, RC. Coronavirus outbreak is a symptom of Gaia's sickness. Ecol Modell [Preprint] 2020 Jun [cited 2020 Apr 27];426: 109075. Available from: https://www.ncbi.nlm.nih. gov/pmc/articles/PMC7158772/pdf/main.pdf doi: https:// doi.org/10.1016/j.ecolmodel.2020.109075

2. Swerdlow DL, Finelli L. Preparation for Possible Sustained Transmission of 2019 Novel Coronavirus: Lessons From Previous Epidemics. Jama [Internet]. 2020 Feb [cited 2020 Apr 23];323(12):1129-30. Available from: https://jamanetwork. com/journals/jama/fullarticle/2761285 doi: https://doi. org/10.1001/jama.2020.1960

3. Jiang L, Broome ME, Ning C. The performance and professionalism of nurses in the fight against the new outbreak of COVID-19 epidemic of Chinese nurses is laudable. Int $J$ Nurs Stud [Preprint]. 2020 Mar [cited 2020 Apr 26]. Available from: https://www.sciencedirect.com/science/article/pii/S0020748920300638?via\%3Dihub doi: https://doi. org/10.1016/j.ijnurstu.2020.103578

4. Aliakbari F, Hammad K, Bahrami M, Aein F. Ethical and legal challenges associated with disaster nursing. Nurs Ethics [Internet]. 2015 Jun [cited $2020 \mathrm{Apr}$ 26];22(4):493-503. Available from: https://journals.sage- pub.com/doi/abs/10.1177/0969733014534877?rfr dat $=c r_{\text {_ }}$ pub\%3Dpubmedgurl_ver $=Z 39.88-2003$ Erfr_ id=ori\%3Arid\%3Acrossref.orgfjournalCode=neja doi: https:// doi.org/10.1177/0969733014534877

5. Choi KR, Jeffers KS, Logsdon MC. Nursing and the Novel Coronavirus: Risks and Responsibilities in a Global Outbreak. J Adv Nurs [Preprint]. 2020 [cited 2020 Apr 26] Available from: https://onlinelibrary.wiley.com/action/ showCitFormats?doi=10.1111\%2Fjan.14369 doi: https://doi. org/10.11l1/jan.14369

6. Chen Q, Liang M, Li Y, Guo J, Fei D, Wang L, et al. Mental health care for medical staff in China during the COVID-19 outbreak. Lancet [Internet]. 2020 Apr [cited 2020 Apr 25];7(4):el5-el6. Available from: https://doi.org/10.1016/S2215-0366(20)30078-X doi: https://doi.org/10.1016/S2215-0366(20)30078-X

7. Huang J, Han M, Luo T, Ren A, Zhou X. Mental Health Survey of 230 Medical Staff in a Tertiary Infectious Disease Hospital for COVID-19. Chinese journal of industrial hygiene and occupational diseases [Preprint]. 2020 Mar [cited 2020 Apr 26];38. Available from: http://rs.yiigle. com/yufabiao/1183760.htm doi: https://doi.org/10.3760/ cma.j.cn121094-20200219-00063 
8. Kang L, Ma S, Chen M, Yang J, Wang Y, Li R, et al. Impact on mental health and perceptions of psychological care among medical and nursing staff in Wuhan during the 2019 novel coronavirus disease outbreak: A cross-sectional study. Brain Behav Immun [Preprint]. 2020 Mar [cited 2020 Apr 22]:(20):30348-2. Available from: https://www.sciencedirect. com/science/article/pii/S0889159120303482?via\%3Dihub doi: https://doi.org/10.1016/j.bbi.2020.03.028

9. Qi J, Xu J, Li B, Huang J, Yang Y, Zhang Z, et al. The Evaluation of Sleep Disturbances for Chinese Frontline Medical Workers under the Outbreak of COVID-19. medRxiv [Preprint]. 2020 Mar [cited 2020 Apr 22]. Available from: https:// www.medrxiv.org/content/10.1101/2020.03.06.20031278v2 doi: https://doi.org/10.1101/2020.03.06.20031278

10. Tavares CQ. Dimensões do cuidado na perspectiva da espiritualidade durante a pandemia pelo novo coronavirus (COVID-19). J Health NPEPS [Internet]. 2020 Apr [cited 2020 Apr 27]; 5(1):1-4. Available from: https://periodicos.unemat.br/index.php/jhnpeps/article/view/4517/3555

11. Sun N, Shi S, Jiao D, Song R, Ma L, Wang H, et al. A Qualitative Study on the Psychological Experience of Caregivers of COVID-19 Patients. Am J Infect Control [Preprint]. 2020 Apr [cited 2020 apr 22]. Available from: https://www. ncbi.nlm.nih.gov/pmc/articles/PMC7141468/ doi: 10.1016/j. ajic. 2020.03 .018

12. Lima CKT, Carvalho PMM, Lima IAAS, Nunes JVAO, Saraiva JS, de Souza RI, et al. The emotional impact of, Coronavirus 2019-nCoV (new Coronavirus disease). Psychiatry Res [Internet]. 2020 May [cited 2020 Apr 23]; 287:112915. Available from: https://www.sciencedirect.com/science/ article/pii/S0165178120305163?via\%3Dihub doi: https://doi. org/10.1016/j.psychres.2020.112915.

13. Lan J, Song Z, Miao X, Li H, Li Y, Dong L, et al. Skin damage among healthcare workers managing coronavirus disease-2019. J Am Acad Dermatol [Internet]. 2020 May [cited 2020 Apr 6]:82(5):1215-1216. Available from: https://www. jaad.org/article/S0190-9622(20)30392-3/pdf doi:https:// doi.org/10.1016/j.jaad.2020.03.014

14. Misra A. Doctors and Healthcare Workers at Frontline of COVID 19 Epidemic: Admiration, A Pat on the Back, and Need for Extreme Caution. Diabetes Metab Syndr [Internet]. 2020 May-June [cited 2020 Apr 26];14(3):255256. Available from: https://www.ncbi.nlm.nih.gov/pmc/ articles/PMC7102612/ doi: https://doi.org/10.1016/j. dsx.2020.03.006
15. Maslow AH. [Motivation and personality]. 2nd ed. New York: Harperand How, 1970. Spanish.

16. Lefreve F, Lefreve AMC. [The collective subject who speaks ]. Interface [Internet]. 2006 Jul/Dec [cited 2020 apr 18];10(20):517-524. Portuguese. Available from: http://www.scielo.br/scielo.php?pid=s1414-328320060 $00200017 \&$ script=sci_abstract\&tlng=pt doi: https://doi. org/10.1590/S1414-32832006000200017.

17. Roy D, Tripathy S, Kar SK, Sharma N, Verma SK, Kaushal V. Study of knowledge, attitude, anxiety \& perceived mental healthcare need in Indian population during COVID-19 pandemic. Asian J Psychiatr [Preprint]. 2020 Jun [cited 2020 apr 10]; 51:102083. Available from: https://www. ncbi.nlm.nih.gov/pmc/articles/PMC7139237/pdf/main. pdf doi: https://doi.org/10.1016/j.ajp.2020.102083

18. Coelho AP, Vieira RAM, Leite MA, Lucas TC. [The impact of the interprofessional learning in ventilation-associated pneumonia: bundles implementation in an intensive care unit]. Enferm Foco [Internet] 2019 [cited 2020 apr 18];10(4):93-100. Portuguese Available from: http://revista.cofen.gov.br/index.php/enfermagem/article/view/2262/611 doi: https://doi.org/10.21675/2357707X.2019.v10.n4.2262

19.Wong SCY, Kwong RTS, Wu TC, Chan JWM, Chu MY, Lee SY, et al. Risk of nosocomial transmission of coronavirus disease 2019: an experience in a general ward setting in Hong Kong. J Hosp Infect [Preprint] 2020 Apr [cited 2020 apr 18];(20):30174-2. Available from: https://www.journalofhospitalinfection.com/ article/S0195-6701(20)30174-2/pdf doi: https://doi. org/10.1016/j.jhin.2020.03.036

20.Gasmi A, Noor S, Tippairote T, Dadar M, Menzel A, Bjørklund G. Individual risk management strategy and potential therapeutic options for the COVID-19 pandemic. Clin Immunol [Preprint]. 2020 Apr [cited 2020 apr 18];108409. Available from: https://www.sciencedirect.com/science/article/pii/ S1521661620302254?via\%3Dihub doi: https://doi. org/10.1016/j.clim.2020.108409

21. Chen Y, Chen L, Deng Q, Zhang G, Wu K, Ni L, et al. The Presence of SARS-CoV-2 RNA in Feces of COVID-19 Patients. J Med Virol [Preprint]. 2020 Apr [cited 2020 apr 22]. Avaliable from: https://onlinelibrary.wiley.com/ doi/full/10.1002/jmv.25825 doi: https://doi.org/10.1002/ jmv. 25825 
22. Jordan K. The Disaster Survivor's Hierarchy of Needs: What Every Disaster Mental Health Worker Should Know. Vistas online [Internet]. 2015 [cited 2020 apr 27];1-7. Avaliable from: https://www.counseling.org/docs/default-source/ vistas/the-disaster-survivor.pdf?sfvrsn=e2db432c_6

23. Yin $X$, Zeng L. A study on the psychological needs of nurses caring for patients with coronavirus disease 2019 from the perspective of the existence, relatedness, and growth theory. Int J Nurs Sci [Preprint]. 2020 Apr [cited 2020 apr 27]. Avaliable from: https://www.ncbi.nlm.nih.gov/ pmc/articles/PMC7128423/pdf/main.pdf doi: https://doi. org/10.1016/j.ijnss.2020.04.002

24. Mohindra R, R R, Suri V, Bhalla A, Singh, SM. Issues relevant to mental health promotion in frontline health care providers managing quarantined/isolated COVID19 patients. Asian J Psychiatr [Internet]. 2020 Jun [cited 2020 apr 18];51:102084. Avaliable from: https://www.sciencedirect.com/science/article/pii/S1876201820301957?via\%3Dihub doi: https://doi.org/10.1016/j.ajp.2020.102084

25. Mattos JCO, Balsanelli AP. [Nurses' leadership in primary health care: na integrative review]. Enferm Foco [Internet]. 2019 [cited 2020 apr 18];10(4):164-171. Portuguese. Available from: http://revista.cofen.gov.br/index.php/enfermagem/ article/view/2618/621 doi: https://doi.org/10.21675/2357707X.2019.v10.n4.2618

26. Chan JF, Yuan S, Kok KH, To KK, Chu H, Yang J, et al. A familial cluster of pneumonia associated with the 2019 novel coronavirus indicating person-to-person transmission: a study of a 286 family cluster. Lancet [Internet]. 2020 Feb [cited 2020 apr 18];395(10223):514-23. Available from: https://www.thelancet.com/journals/lancet/article/PIIS0140-6736(20)30154-9/fulltext doi: https://doi. org/10.1016/s0140-287

27. Li Q, Guan X, Wu P, Wang X, Zhou L, Tong Y, et al. Early transmission dynamics in Wuhan, China, of novel coronavirus infected pneumonia. N Eng J Med [Internet]. 2020 Mar [cited 2020 apr 23];382(13):1199-1207. Available from: https://www.nejm.org/doi/full/10.1056/NEJMoa2001316 doi: https://doi.org/10.1056/NEJMoa2001316

28. Wang J, Zhao S, Liu M, Zhao Z, Xu Y, Wang P, et al. ACE2 expression by colonic epithelial cells is associated with viral infection immunity and energy metabolismo medRxiv [Preprint]. 2020 [cited 2020 apr 18];1-13. Available from: https:// www.medrxiv.org/content/10.1101/2020.02.05.20020545vl doi: https://doi.org/10.1101/2020.02.05.20020545
29. Xia J, Tong J, Liu M, Shen Y, Guo D. Evaluation of coronavirus in tears and conjunctival secretions of patients with SARS-CoV-2 infection. J Med Virol [Preprint]. 2020 Feb [cited 2020 apr 18];1-6. Available from: https://onlinelibrary.wiley. com/action/showCitFormats?doi=10.1002\%2Fjmv. 25725 doi: https://doi.org/10.1002/jmv.25725

30. Xiao F, Tang M, Zheng X, Li C, He J, Hong Z, et al. Evidence for gastrointestinal infection of SARS-CoV-2. Gastroenterol [Internet]. 2020 May [cited 2020 apr 18];125(6):1831-1833e. Available from: https://www.ncbi.nlm. nih.gov/pubmed/32142773 doi: https://doi.org/10.1053/j. gastro.2020.02.055

31. Goldschmidt K. The COVID-19 pandemic: Technology use to support the wellbeing of children. $J$ Pediatr Nurs [Preprint]. 2020 Apr [cited 2020 apr 26];(20):30269-4. Available from: https://www.pediatricnursing.org/article/ S0882-5963(20)30269-4/pdf doi: https://doi.org/10.1016/j. pedn.2020.04.013

32. To KK, Tsang OT, Chik-Yan Yip C, Chan KH, Wu TC, Chan JMC, et al. Consistent detection of 2019 novel coronavirus in saliva. Clin Infect Dis [Preprint]. 2020 Feb [cited 2020 apr 25]. Available from: https://academic.oup.com/ cid/advance-article/doi/10.1093/cid/ciaal49/5734265 doi: https://doi.org/10.1093/cid/ciaal49

33. Patri A, Gallo L, Guarino M, Fabbrocini G. Sexual transmission of severe acute respiratory syndrome coronavirus 2 (SARS-CoV-2): A new possible route of infection? J Am Acad Dermatol [Preprint]. 2020 Apr [cited 2020 apr 18]. Available from: https://linkinghub.elsevier.com/retrieve/ pii/S0190-9622(20)30521-1 doi: https://doi.org/10.1016/j. jaad.2020.03.098

34. Rocha JC, Ruiz VM. [Quality of life and work ability of physicians and nurse workers from hospitals]. Braz J of Develop [Internet]. 2019 [cited 2020 apr 27];5(11):23546-23576. Portuguese. Available from: http://www.brazilianjournals. com/index.php/BRJD/article/view/4386/4112 doi: https:// doi.org/10.34117/bjdv5n11-065

35. Gender NV, Peterman A, Potts A, O'Donnell M. Thompson K, Shah N, et al. COVID-19: Reducing the risk of infection might increase the risk of intimate partner violence. EClinicalMedicine [Preprint]. 2020 Apr [cited 2020 apr 18]. Available from: https://www. thelancet.com/pdfs/journals/eclinm/PIIS25895370(20)30092-4.pdf doi: https://doi.org/10.1016/j. eclinm.2020.100348 